\title{
El uso del Ambiente Virtual de Aprendizaje entre el profesorado de educación superior: un análisis de género.
}

\section{Use of the Virtual Learning Environment among Higher Education Teaching Staff: A Gender Analysis.}

\author{
Annachiara Del Prete \\ Centro de Investigación en Educación Inclusiva, Pontificia Universidad Católica de Valparaíso (PUCV). Valparaíso, \\ Chile. \\ annachiara.delprete@gmail.com \\ Julio Cabero Almenara \\ Universidad de Sevilla. Sevilla, España \\ cabero@us.es
}

\section{Resumen}

El uso masivo de la tecnología, en la vida cotidiana y en la educación en particular, está teniendo siempre más influencias en la autopercepción, por parte de los/as docentes, de habilidades de uso de las TIC, así como en sus competencias digitales. El propósito del estudio ha sido analizar si se presentan diferencias de género en el uso pedagógico de la plataforma Moodle, encuestando una población de 640 docentes de una institución de educación superior técnico profesional de Chile. El estudio ha sido realizado a través del análisis estadístico basado en la prueba de significación de hipótesis nula y la variable género ha sido analizada de forma descontextualizada, sin tomar en consideración las interacciones que se puedan dar con otras variables. Entre los resultados principales se destaca la evidencia de que no se presentan diferencias significativas entre profesores y profesoras el uso didáctico de la plataforma Moodle, así como en la auto calificación del dominio de la misma.

Palabras claves: brecha digital de género; Ambientes Virtual de Aprendizaje; Educación Superior; competencias digitales.

\begin{abstract}
The widespread use of technology in daily life, and particularly in education, increasingly influences both the self-perception of Information and Communication Technology skills and teachers' digital competence. This study aims to analyze if there are gender differences in the pedagogical use of the Moodle platform, by conducting surveys among a population of 640 teachers from a technical professional higher education institution in Chile. The study of gender differences has been conducted through a statistical analysis based on a significance test of null hypothesis and the gender variable has been analyzed in a decontextualized manner, without taking into account the interactions that they could have with other variables. Among the primary results, evidence shows that there are no significant differences between female and male teachers with regard to the educational use of the Moodle platform as well as selfqualification with respect to proficiency on the platform.
\end{abstract}

Keywords: Digital Gender Gap; Digital Competences; Learning management systems; LMS; Pedagogy; Higher education.

RED. Revista de Educación a Distancia. Núm. 62, Vol. 20. Artíc. 04, 31-03-2020

DOI: http://dx.doi.org/10.6018/red.400061 


\section{Introducción}

Como resultado de la presencia de las tecnologías de la información y la comunicación (TIC) en un nivel siempre más integrado a nuestro sistema de vida, también la educación está experimentando cambios que, según los contextos políticos y sociales de cada país, siguen diferentes paradigmas y modelos para sus innovaciones y para la formulación de reformas necesarias a garantizar su adopción y uso (Chiu \& Linn, 2012). Para el caso particular de la educación superior, como elemento destacable, encontramos que, en las últimas dos décadas, el uso de Ambientes Virtuales de Aprendizajes (AVA) (por Ambiente Virtual de Aprendizaje entendemos el conjunto de entornos de interacción, sincrónica y asincrónica, donde, con base en un programa curricular, se lleva a cabo el proceso enseñanza-aprendizaje, a través de un sistema de administración de aprendizaje), ha vivido una significativa extensión (Bidarian \& Davoudi 2011). Entre los motivos que pueden explicar este fenómeno, se identifica la agilidad en la sistematización del trabajo de gestión y planificación docente, que ha hecho que, en numerosas instituciones superiores, el uso de los AVA, se haya vuelto obligatorio para el quehacer diario del profesorado (Krause et al. 2017). Junto con esto encontramos la contribución, que los AVA han demostrado aportar, al mejoramiento de la comunicación entre estudiantes y docentes; en el diseño de actividades prácticas de aprendizaje y de evaluación facilitando, de tal manera, el uso de modelos de enseñanza-aprendizaje centrados en el estudiante, promoviendo el trabajo colaborativo y el intercambio de información (Rodrìguez et al. 2016). No por último, encontramos una siempre más fuerte competencia, entre las instituciones de educación superior, para atraer estudiantes y satisfacer sus necesidades y objetivos educativos (Clark \& Meyer, 2011) y para apoyar la entrega presencial y remota de los cursos sin las limitaciones de tiempo y distancia, hecho que ha impulsado de manera importante el uso de sistemas e-learning y blended learnig (Aristovnik et al. 2017).

Sin embargo, no obstante, esta evidente expansión de la adopción de tecnologías, un contexto rico de soportes tecnológico no es suficiente para garantizar el uso y la implementación de las TIC en la educación (Sosa, Salinas \& De Benito, 2018), así como no es suficiente para garantizar la máxima explotación de los AVA en la práctica docente (Raman, Rozalina Khalid \& Rizuan 2014).

De hecho, los estudios nos informan que la adopción y uso de las TIC en los procesos educativos, todavía se enfrenta una cierta resistencia por parte del profesorado (BuabengAndoh, 2012; Kafyulilo, Fisser \& Voogt, 2016; Teo 2011). Las causas de esta resistencia son diversas y varían entre la dificultad de acceso a los recursos, la falta de competencia técnica y pedagógica, la falta de materiales curriculares, y la falta de apoyo técnico y formativo (Casquero, García \& González, 2014). Pero, entre todos los factores evidenciados, hay que destacar un aspecto en particular, el cual juega un rol importante para garantizar la incorporación y el uso de las TIC en la sala de clase y este es la aceptación, que tanto el alumnado como el profesorado presentan respecto de las TIC (Lohbeck, Hagenauer, \& Frenzel 2018; Krause et al. 2017). A tal propósito Radovan y Kristl (2017) han señalado, recientemente, que los docentes presentan un alto grado de aceptación de los AVA y que este

El uso del Ambiente Virtual de Aprendizaje entre el profesorado de educación superior: un análisis de género. Annachiara Del Prete y Julio Cabero.

Página 2 de 20 
grado de aceptación está fuertemente relacionado con la percepción de utilidad que tiene la aplicación tecnológica.

Sobre esta acepción de la tecnología pueden incidir variables de diversos tipos, como las variables personales, de comportamiento y de entorno (Dusick,1998). Las variables personales y de comportamiento se refieren al conocimiento, la competencia, la actitud, la percepción, las creencias y el compromiso, mientras que las variables de entorno se refieren a las instalaciones, el equipamiento y el soporte. Otros estudios han evidenciado los efectos de variables externas, entre las cuales se encuentra el disfrute percibido en el uso de estas herramientas en el proceso de enseñanza (Teo, 2011), la presencia de condiciones facilitadoras (Terzis \& Economides, 2011), la influencia social (Moran, Hawkes \& El Gayar, 2010; Terzis \& Economides, 2011), el concepto de autoeficacia (Ahmad et al. 2010; Chen, 2010;), la experiencia en el uso de TIC y capacitación (Asiri 2012; Mahmud \& Ismail, 2010); y no por último las variables demográficas como el género y la edad (Venkatesh \& Morris, 2000).

En particular el género ha resultado ser una variable particularmente influyente para medir el grado de aceptación y para determinar la intención de uso de las TIC en la enseñanza (Asiri, 2012; Cabero, Sampedro y Gallego, 2016; Tsai y Tsai 2010; Wong, Teo \& Russo, 2012), tanto que las investigaciones sobre las posibles diferencias de género en el uso y adopción de las tecnología en el contexto educativo, se ha disparado desde principios de siglo (United Nations 2014; OCDE, 2010; Tüfekçi, 2008; Vázquez-Cano, Meneses, \& GarcíaGarzón, 2017).

De hecho podemos encontrar los resultados de diferentes investigaciones donde se ha puesto de manifiesto la existencia de una brecha digital entre los profesores y las profesoras, brecha que incide, en mayor mesura, en la percepción de competencias tecnológicas y en las pedagógicas con el uso de las TIC (Suárez, Almerich, Gargallo \& Aliaga 2013) y en la tendencia a una utilización diferentes de las tecnologías (Cantón, Cañón y Grande, 2017). En particular, con base a dichos estudios, las profesoras presentarían percepciones de menos utilidad y mostrarían una menor intención de uso de sistemas tecnológicos como el e-learning (Ong y Lai, 2006; Romero, Cabero \& Barroso, 2016). Al mismo tiempo las profesoras presentarían menor frecuencia de utilización de las TIC en el aula, hecho que se confirma incluso teniendo los dos colectivos (profesoras y profesores), el mismo nivel de competencias digitales (Vekiri \& Chronaki, 2008).

A raíz de estas evidencias, diferentes investigaciones han analizado con atención, los factores que influyen en la aceptación de las mujeres en la adopción de las TIC y el factor que ha resultado ser determinante es el concepto de autoeficacia, concepto que se refiere a la opinión que una persona tiene sobre sus propias habilidades para desarrollarse con éxito en un campo particular (Bandura, 1977; Zeldin y Pajares, 2000) y es un mecanismo mediador entre el conocimiento y la acción, vinculándose estrechamente con la autorregulación de las motivaciones y decisiones (Rossi y Barajas, 2015). Diferentes investigaciones indican que las mujeres presentan menor percepción autoeficacias, o sea de habilidades de manejo de la tecnología respecto a los hombres (Ong y Lai 2006; Sieverding y Koch 2009; Koch, Muller y Sieverding 2008).

El uso del Ambiente Virtual de Aprendizaje entre el profesorado de educación superior: un análisis de género. Annachiara Del Prete y Julio Cabero.

Página 3 de 20 
Pero si la autoeficacia es un concepto fundamental para explicar los procesos relacionados con las aspiraciones, las elecciones académico-profesionales y la adopción de las TIC en la práctica didáctica, no hay que perder de vista la diferencia encontrada entre una autoeficacia aparente (declarada) y otra real (decisoria, creencias personales respecto a las habilidades y capacidades) (Rossi y Barajas, 2015), en las cuales, normalmente de forma inconsciente, juegan un importante papel los estereotipos y roles de género que afloran en las decisiones académicas y profesionales (Bandura, Barbaranelli, Caprara y Pastorelli, 2001; Bleeker y Jacobs, 2004). Los estereotipos y las variables contextuales explicarían porque las mujeres tienden a subestimar sus habilidades en internet y en el manejo de las TIC, si bien en la práctica no se arrojan diferencias significativas de habilidades técnicas y pedagógicas entre hombres y mujeres en el uso de las TIC en el aula (Almerich et al. 2005; Almerich et al. 2011; Chou, Wu \& Chen, 2011; Law, Pelgrum, \& Plomp, 2008; Ríos, Gómez \& Priscilla, 2018; Terzis \& Economides, 2011; Vekiri \& Chronaki 2008).

Interesante, a tal propósito, es revisar el estudio de Schumacher \& Morahan-Martin (2001) en el cual se analizó la evolución de las experiencias, las destrezas y actitudes hacia internet a lo largo de una década, a través dos generaciones de estudiantes 1989/90 y 1997. Los resultados demostraron como la facilidad de acceso y las habilidades de uso estaban altamente intercorrelacionados y ambas variables predijeron habilidades y experiencias en Internet. Si bien se evidenciaron diferencias de habilidades entre hombres y mujeres, sobretodo en la generación del 89/90, comparando los resultados con los de la generación del 1997 se demostró que a medida que se daban facilidades de acceso y apoyo al uso de las TIC, las competencias de las/os estudiantes aumentaban y las diferencias de género decrecían.

Con base a este último hallazgo se podría sostener que las diferencias de género en la aceptación, en la autoeficacia y en el uso de las TIC responde a unas series de inequidades socioculturales que impactan en la percepción de habilidades y competencias y por ende en la acceso a la formación y en el sesgo de determinadas carreras académicas; muy incidentes también resultan ser los estereotipos de género, la poca formación o, como en un principio ocurrió por la navegación en red, la prevalencia de contenido sesgado (Pohl, 1997; Weiser, 2000), prevalencia explicada por la relación entre el poder y la informática, ámbitos de influencias prevalentemente masculino (Grundy 1996; Turkle 1995; Sussman \& Tyson, 2000). Sin embargo, a medida que estas mismas desigualdades y diferencias van desapareciendo, la brecha de género en el uso de TIC también lo hace. De hecho, ya se puede observar como estudios actuales confirman y refutan la existencia de diferencias entre hombres y mujeres en el uso de la tecnología, según el contexto y la perspectiva de análisis que se aplica (Chou, Wu, \& Chen 2011; Güdüz e İşman, 2018; Lohbeck, Hagenauer, \& Frenzel, 2018; Ríos, Gómez y Priscilla, 2018; Vázquez-Cano, Meneses, \& García-Garzón, 2017).

Tomamos el caso de la investigación de Raman, Rozalina Khalid \& Rizuan (2014) en la cual se analizó el uso de la plataforma Moodle en una muestra de 65 estudiantes universitarios malayos, el estudio no arrojó diferencias entre hombres y mujeres. Rodríguez Espinosa, et al. (2016), analizaron la alfabetización informática del profesorado y el uso de un sistema de gestión del aprendizaje (LMS) en la educación superior y se detectó mayor conocimiento de las mujeres sobre el uso de las TIC en la docencia. Resultados similares fueron encontrados por Suri \& Sharma (2013) en India, y otros estudios han demostrado que las mujeres tienen

El uso del Ambiente Virtual de Aprendizaje entre el profesorado de educación superior: un análisis de género. Annachiara Del Prete y Julio Cabero.

Página 4 de 20 
una mayor predisposición hacía el uso de la tecnología para la enseñanza respecto a los docentes. Egbo, Chinwe, Ikechukwu \& Onwumere (2011) estudiaron a 415 estudiantes preuniversitarios en Nigeria y descubrieron que las mujeres estaban más inclinadas hacia dispositivos tecnológicos que sus homólogos masculinos.

Por lo reportado de la literatura, se puede observar como a medida que las TIC han ido permeando las tareas diarias de las personas y que las instituciones educativas, en el caso concreto las Institución de Educación Superior, las han ido adoptando para tareas de gestión y para la planificación docente, como es el caso de Ambientes Virtuales de Aprendizaje, el uso de las TIC se ha vuelto una tarea más del quehacer docente. Esto ha tenido un impacto positivo sobre la diferencias de género que se iban presentando en las habilidades del profesorado, de hecho estudios recientes indican una nivelación de las competencias digitales de profesoras y profesores y una atenuación en las diferencias del concepto de autoeficacias, presentando las mujeres mayor aceptación de las TIC y mejor percepción de sus habilidades digitales y pedagógicas (Chou, Wu, \& Chen, 2011; Teo, 2010; Schumacher \& Morahan-Martin, 2001). Al mismo tiempo el dato que más se destaca es, que al parecer, la tendencia de uso de las herramientas TIC para la enseñanza y el uso pedagógico de los ambientes de aprendizaje esté cambiando a favor de las mujeres; siempre más estudios registran, de hecho, una actitud más activa de las profesoras y de las estudiantes, en el proceso de aprendizaje a través de las tecnología, respecto a sus homólogos varones (GonzálezGómez et al 2012; Egbo, Chinwe, Ikechukwu, \& Onwumere 2011; Rodríguez, Restrepo \& Aranzazu, 2014; Suri \& Sharma 2013). Y sobre ello queremos profundizar en la presente investigación en el caso concreto de una Universidad chilena expandida por todo el país.

\section{Método}

\section{Objetivos}

El objetivo general que persigue el estudio, ha sido conocer si existen diferencias en la utilización del "Ambiente de Aprendizaje de la Universidad Tecnológica de Chile INACAP" (AAI), es decir en el entorno virtual Moodle creado por la Institución, y las diferentes herramientas en él incorporadas en la formación virtual, en función del género de los docentes. De este objetivo general se desglosan una serie de preguntas: referidas a si había diferencias significativas entre los profesores y las profesoras respecto a:

- ¿Hay diferencias significativas en la utilización del ambiente de aprendizaje de INACAP (AAI) entre los profesores y profesoras?

- ¿Los motivos de no utilización de la plataforma (obstáculos) dependen del género del docente?

- ¿La valoración efectuada de diferentes aspectos del AAI, vienen determinadas por el género del docente?

- ¿El dominio técnico, manejo didáctico y frecuencia de utilización de diferentes herramientas utilizadas en la formación virtual difiere en función del género del docente?

- ¿Los profesores y las profesoras realizan actividades diferentes en el AAI de INACAP?

- ¿Utilizan los profesores y las profesoras los mismos tipos de materiales en el entorno AAI de INACAP?

El uso del Ambiente Virtual de Aprendizaje entre el profesorado de educación superior: un análisis de género. Annachiara Del Prete y Julio Cabero.

Página 5 de 20 
- ¿El dominio declarado de AAI por los docentes viene determinado por su género?

- Y el trabajo realizado en el AAI.

Hay que señalar que para el análisis de los siguientes objetivos utilizaremos diferentes pruebas estadísticas, fundamentalmente estadística descriptiva, estadístico de Levene para determinar el valor $\mathrm{t}$ de student para muestras independientes que utilizaremos. También aplicaremos el estadístico alfa de Cronbach para la determinación del índice de fiabilidad del instrumento.

\section{Población y muestra de la investigación}

El grupo de docentes de la institución INACAP sujetos de estudio, durante el curso académico 2017-18 fueron 5234, de los cuales cumplimentaron correctamente el cuestionario 640 , lo que supone el 12,23\% de la población; ello implicaba que se trabajó con una muestra cuyo tamaño supera los 358 que serían los mínimos necesarios para obtener un nivel de confianza del $95 \%$ en el estudio, por el tamaño muestral. La muestra de la investigación es del tipo no probabilística, de conveniencia o causal (Alamino, 2006; Sabariego, 2012), que viene determinada por la facilidad de acceso que el investigador tiene a los sujetos que la conforman.

De los 640, 231 (36,09\%) eran hombres y 409 (63,91\%) mujeres; trabajando la gran mayoría ( $\mathrm{f}=565,82,12 \%)$ en la modalidad presencial, seguidos a gran distancia de $16(2,52)$ que lo hacían en la modalidad "on-line". Y por lo que se refiere a las jornadas donde trabajaban: 47 $(7,46 \%)$ lo hacían en la vespertina, $187(29,68 \%)$ en la diurna y 396 (f=62,86\%) en ambas; indicar que 3 docentes no cumplimentaron esta respuesta. Por lo que se refiere a INACAP indicar que es la Institución de Educación Superior más grande y diversa de Chile, que imparte docencia en todo el país a través de 26 sedes.

\section{Instrumentos de recogida de información.}

El instrumento de recogida de información estaba compuesto por 21 ítems de diferente tipología: cumplimentación, respuesta tipo Likert, opciones de respuestas dicotómicas y autovaloración numérica. El instrumento recoge información de diferentes aspectos: características personales del encuestado, creencias de los docentes sobre la forma de enseñar, frecuencia de usos del AAI, dominio técnico, didáctico y frecuencia de uso de AAI, actividades y estrategias que son desarrolladas por el docente con el apoyo de AAI, frecuencia de utilización de las herramientas que incorpora el AAI, y trabajo de la asignatura con el apoyo del AAI.

La diversidad de tipología del cuestionario nos ha llevado a analizar su índice de fiabilidad según las diferentes tipologías de preguntas, en la tabla $\mathrm{n}^{\circ} 1$ se presentan los valores obtenidos con el estadístico alfa de Cronbach.

Tabla 1 : Índice de Fiabilidad según tipología de pregunta

\begin{tabular}{l|c}
\hline \multicolumn{1}{c|}{ Dimensión } & Alfa de Cronbach \\
\hline Creencia de los docentes sobre su forma de enseñar & 0,890 \\
\hline Motivos por los cuales no utilizó el AAI & 0,932 \\
\hline
\end{tabular}

El uso del Ambiente Virtual de Aprendizaje entre el profesorado de educación superior: un análisis de género. Annachiara Del Prete y Julio Cabero.

Página 6 de 20 
RED. Revista de Educación a Distancia. Núm. 62 Vol. 20. Artíc. 04, 31-03-2020

DOI: http://dx.doi.org/10.6018/red.400061

\begin{tabular}{l|l}
\hline Dominio técnico del AAI & 0,918 \\
\hline Dominio didáctico del AAI & 0,915 \\
\hline Frecuencia de uso del AAI & 0,872 \\
\hline Actividades y estrategias desarrolladas con apoyo de AAI & 0,925 \\
\hline Recursos de aprendizaje que se incorporan en el AAI & 0,868 \\
\hline Sobre el trabajo de tu asignatura con apoyo de AAI & 0,927 \\
\hline
\end{tabular}

Los valores obtenidos con el alfa de Cronbach, de acuerdo con la propuesta de O’Dwyer y Bernauer (2014), nos sugieren que el instrumento en sus diferentes dimensiones posee unos niveles altos de fiabilidad. Señalar que también se efectuó la correlación ítem-total, por si la eliminación de algún ítem ampliaba el nivel global de fiabilidad. Hecho que no fue necesario.

\section{Resultados}

De acuerdo con los objetivos planteados en la investigación, los resultados más relevantes son los siguientes:

\section{Utilización del ambiente de aprendizaje (AAI) de INACAP.}

Con el objeto de analizar si había diferencias estadísticas significativas en función del género de los y las docentes, y si en el último año habían utilizado el AAI, se formularon las siguientes hipótesis:

H0 (hipótesis nula): No hay diferencias estadísticamente significativas en función del género en haber utilizado la plataforma AAI de INACAP en el último año en función del género del docente, con un riesgo alfa de equivocarnos del 0,05.

H1 (hipótesis alternativa): Si hay diferencias estadísticamente significativas en función del género del docente, en haber utilizado la plataforma AAI de INACAP en el último año en función del género del docente, con un riesgo alfa de equivocarnos del 0,05.

Hay que señalar que, por género, 7 hombre y 49 mujeres indicaron que no habían utilizado la plataforma AAI y 224 hombres y 360 mujeres que sí.

Para contrastar las hipótesis anteriores aplicamos la Q de Yule (Bishop, Fienberg y Holland, 2007), obteniendo el valor de $-0,627$, con un valor de $Z=-2,498$ y nivel de significación de $\mathrm{p}<0,006$. Valores que nos permiten rechazar la $\mathrm{H} 0$ a un nivel de significación de $\mathrm{p}<0,01$; en consecuencia, podemos concluir que el género de los docentes repercute en la frecuencia de utilización de del AAI en el último año. El valor negativo nos indica que en proporción los profesores tienden a utilizarlo más que las profesoras. Podríamos decir que, aunque en frecuencia absoluta las profesoras tienden a utilizar más el AAI, en proporción el "Si" se asocia a los profesores y el "No" a las profesoras. Si bien en términos absolutos las frecuencias de profesoras que lo utilizan es superior a los profesores.

\section{Motivos no utilización de la plataforma (obstáculos).}

Preguntado el profesorado por los motivos que los llevaban a no utilizar la plataforma, las puntuaciones medias alcanzadas se presentan en la tabla $\mathrm{n}^{\circ} 2$. Para una correcta interpretación de los valores obtenidos se debe tener en cuenta que el intervalo de respuesta iba de 1 (muy poca importancia) a 10 (muchísima importancia).

El uso del Ambiente Virtual de Aprendizaje entre el profesorado de educación superior: un análisis de género. Annachiara Del Prete y Julio Cabero.

Página 7 de 20 
RED. Revista de Educación a Distancia. Núm. 62 Vol. 20. Artíc. 04, 31-03-2020

DOI: http://dx.doi.org/10.6018/red.400061

Tabla 2 Motivo de no utilización del AAI

\begin{tabular}{|c|c|c|c|c|}
\hline & Sexo & $\mathrm{N}$ & $\mathrm{M}$ & D.tp. \\
\hline \multirow[t]{2}{*}{ Desconozco su utilización técnica } & $\mathrm{H}$ & 22 & 4,91 & 3,365 \\
\hline & $\mathrm{M}$ & 48 & 4,35 & 3,336 \\
\hline \multirow[t]{2}{*}{ Desconozco su utilización educativa } & $\mathrm{H}$ & 20 & 5,30 & 3,262 \\
\hline & $\mathrm{M}$ & 48 & 4,23 & 3,309 \\
\hline \multirow[t]{2}{*}{ Mi experiencia como docente me indica que no les interesa a los estudiantes } & $\mathrm{H}$ & 20 & 5,35 & 3,200 \\
\hline & M & 50 & 4,86 & 3,110 \\
\hline \multirow[t]{2}{*}{ No creo que sea una estrategia de enseñanza útil } & $\mathrm{H}$ & 20 & 4,85 & 3,407 \\
\hline & $\mathrm{M}$ & 48 & 4,79 & 3,222 \\
\hline \multirow[t]{2}{*}{ No se adapta a los contenidos que imparto. } & $\mathrm{H}$ & 21 & 5,19 & 2,732 \\
\hline & M & 49 & 4,78 & 3,255 \\
\hline \multirow[t]{2}{*}{ No he recibido apoyo de la institución para utilizarlo } & $\mathrm{H}$ & 19 & 4,58 & 2,545 \\
\hline & $\mathrm{M}$ & 47 & 4,64 & 3,442 \\
\hline \multirow[t]{2}{*}{ Me exige invertir mucho tiempo } & $\mathrm{H}$ & 20 & 5,55 & 3,000 \\
\hline & $\mathrm{M}$ & 50 & 5,40 & 3,169 \\
\hline \multirow[t]{2}{*}{ Los alumnos no aprenden con ella } & $\mathrm{H}$ & 20 & 5,25 & 2,770 \\
\hline & $\mathrm{M}$ & 49 & 4,51 & 3,209 \\
\hline \multirow[t]{2}{*}{ No tengo materiales educativos } & $\mathrm{H}$ & 18 & 4,39 & 2,933 \\
\hline & $\mathrm{M}$ & 48 & 4,46 & 3,248 \\
\hline \multirow[t]{2}{*}{ Los otros compañeros que imparten las asignaturas no lo utilizan } & $\mathrm{H}$ & 18 & 4,94 & 3,262 \\
\hline & $\mathrm{M}$ & 44 & 4,16 & 2,996 \\
\hline \multirow{2}{*}{$\begin{array}{l}\text { Creo que no sería capaz de usar el Moodle de manera efectiva en mi labor como } \\
\text { docente }\end{array}$} & $\mathrm{H}$ & 16 & 4,31 & 3,177 \\
\hline & $\mathrm{M}$ & 45 & 4,49 & 3,355 \\
\hline \multirow{2}{*}{$\begin{array}{l}\text { Soy reticente con respecto al uso del Moodle porque restringe la interacción social } \\
\text { y aísla a la gente. }\end{array}$} & $\mathrm{H}$ & 18 & 4,56 & 3,072 \\
\hline & $\mathrm{M}$ & 44 & 3,95 & 3,227 \\
\hline \multirow[t]{2}{*}{ Me incomoda que mis estudiantes sean más hábiles en el uso del Moodle. } & $\mathrm{H}$ & 15 & 2,87 & 2,446 \\
\hline & $\mathrm{M}$ & 43 & 3,05 & 3,055 \\
\hline \multirow{2}{*}{$\begin{array}{l}\text { Creo que el Moodle es útil sólo para trabajo de administración y planificación } \\
\text { docente (pruebas, planificación, etc) }\end{array}$} & $\mathrm{H}$ & 20 & 7,00 & 2,884 \\
\hline & $\mathrm{M}$ & 48 & 4,40 & 3,120 \\
\hline \multirow{2}{*}{$\begin{array}{l}\text { Me inquieta la idea de que el Moodle reduzca el rol del profesor, y sea negativo } \\
\text { para la educación de los estudiantes. }\end{array}$} & $\mathrm{H}$ & 18 & 5,00 & 3,343 \\
\hline & $\mathrm{M}$ & 47 & 3,98 & 3,166 \\
\hline \multirow{2}{*}{$\begin{array}{l}\text { Debería usar el Moodle en mis asignaturas pero no sé cómo organizar y gestionar } \\
\text { el aprendizaje de mis estudiantes de esa manera }\end{array}$} & $\mathrm{H}$ & 19 & 4,89 & 3,107 \\
\hline & $\mathrm{M}$ & 45 & 4,91 & 3,225 \\
\hline
\end{tabular}

El análisis de los valores de la tabla 2, nos señala en primer lugar que al observar las desviaciones típicas, en la gran diversidad de contestaciones encontradas, no ha existido una homogeneidad en las respuestas. Por otra parte, los valores no tienden a situarse por encima de la puntuación media de la escala de 5, lo que nos indica que las y los docentes no han tendido a considerar de cierta importancia las diferentes opciones, lo que podría sugerir cierta limitación del instrumento en lo referido a la validez de contenido.

Finalmente indicar que los obstáculos fundamentales que se han percibido, independientemente del género del profesorado, han sido: "Mi experiencia como docente me indica que no les interesa a los estudiantes", y "Me exige invertir mucho tiempo", "Los alumnos no aprenden con ella"; hecho que pudiera deberse a la preocupación que han mostrado por su dominio técnico y educativo de la plataforma.

Nos gustaría destacar las bajas puntuaciones medias obtenidas en el ítem: "Me incomoda que mis estudiantes sean más hábiles en el uso del Moodle" (2,87-hombres y 3,05-mujeres); hecho que confirma que esa teoría de diferenciación entre docentes y discentes, como

El uso del Ambiente Virtual de Aprendizaje entre el profesorado de educación superior: un análisis de género. Annachiara Del Prete y Julio Cabero.

Página 8 de 20 
emigrantes y nativos digitales, donde a los primeros se les considera con un bajo dominio en su capacitación tecnológica no es tan correcta, como está viniendo a indicar últimamente diferentes investigaciones (Mas, 2017; Rodríguez-García, Raso y Ruiz-Palmero, 2019)

Con el objeto de analizar si había diferencias significativas, en función del género, en la percepción de los diferentes obstáculos que repercutían la no utilización del ambiente de AAI, aplicamos el estadístico $\mathrm{t}$ de Student para muestras independientes, realizando previamente la prueba de Levene para el contraste de la igualdad de las varianzas, para que en función de dicho valor, determinar el valor " $\mathrm{t}$ "a adoptar (en la tabla $\mathrm{n}^{\mathrm{o}}$ 3, se presentan los valores " $t$ " obtenidos y el nivel de significación que nos permitía aceptar o rechazar la H0 formulada, referida a la no existencia de diferencias significativas).

Tabla $3 t$ de Student para lo motivos por el no utilizo del AAI

\begin{tabular}{|c|c|c|c|c|c|c|}
\hline & & $\mathrm{F}$ & Sig & $\mathrm{t}$ & g1 & Sig \\
\hline \multirow[t]{2}{*}{ Desconozco su utilización técnica } & A & \multirow[t]{2}{*}{,061 } &, 805 & ,644 & 68 &, 522 \\
\hline & B & & & ,642 & 40,503 &, 524 \\
\hline \multirow[t]{2}{*}{ Desconozco su utilización educativa } & A & ,025 & 874 & 1,221 & 66 & ,226 \\
\hline & B & & & 1,228 & 36,096 & ,227 \\
\hline \multirow{2}{*}{$\begin{array}{l}\text { Mi experiencia como docente me indica que no les } \\
\text { interesa a los estudiantes }\end{array}$} & A & ,000 & ,996 & ,591 & 68 & ,557 \\
\hline & $\mathrm{B}$ & & &, 583 & 34,181 &, 563 \\
\hline \multirow[t]{2}{*}{ No creo que sea una estrategia de enseñanza útil } & A & ,055 & 816 & ,067 & 66 & ,947 \\
\hline & B & & & ,065 & 33,896 & ,948 \\
\hline \multirow[t]{2}{*}{ No se adapta a los contenidos que imparto. } & A & 1,315 & 256 &, 512 & 68 & ,611 \\
\hline & B & & & 549 & 44,829 & ,586 \\
\hline \multirow[t]{2}{*}{ No he recibido apoyo de la institución para utilizarlo } & A & 2,998 & ,088 &,- 068 & 64 & ,946 \\
\hline & B & & &,- 077 & 44,849 & ,939 \\
\hline \multirow[t]{2}{*}{ Me exige invertir mucho tiempo } & A & , 148 & ,702 & ,182 & 68 &, 856 \\
\hline & B & & & ,186 & 36,898 &, 853 \\
\hline \multirow[t]{2}{*}{ Los alumnos no aprenden con ella } & A & 1,208 & 276 & ,902 & 67 & ,370 \\
\hline & B & & & ,960 & 40,688 & ,343 \\
\hline \multirow[t]{2}{*}{ No tengo materiales educativos } & A & ,504 & ,480 & $\begin{array}{l}, 079 \\
\end{array}$ & 64 &, 937 \\
\hline & B & & &,- 083 & 33,655 & ,934 \\
\hline \multirow{2}{*}{$\begin{array}{l}\text { Los otros compañeros que imparten las asignaturas no } \\
\text { lo utilizan }\end{array}$} & A & ,007 & ,933 & ,913 & 60 & ,365 \\
\hline & B & & & 881 & 29,368 & ,386 \\
\hline \multirow{2}{*}{$\begin{array}{l}\text { Creo que no sería capaz de usar el Moodle de manera } \\
\text { efectiva en mi labor como docente }\end{array}$} & A &, 178 & ,675 &,- 183 & 59 &, 855 \\
\hline & $\mathrm{B}$ & & &,- 188 & 27,762 &, 852 \\
\hline \multirow{2}{*}{$\begin{array}{l}\text { Soy reticente con respecto al uso del Moodle porque } \\
\text { restringe la interacción social y aísla a la gente. }\end{array}$} & A & , 370 & ,545 &, 675 & 60 &, 503 \\
\hline & B & & & ,689 & 33,146 & ,496 \\
\hline \multirow{2}{*}{$\begin{array}{l}\text { Me incomoda que mis estudiantes sean más hábiles } \\
\text { en el uso del Moodle. }\end{array}$} & A & 1,359 & ,249 &,- 206 & 56 &, 838 \\
\hline & B & & &,- 229 & 30,385 & ,820 \\
\hline \multirow{2}{*}{$\begin{array}{l}\text { Creo que el Moodle es útil sólo para trabajo de } \\
\text { administración y planificación docente (pruebas, } \\
\text { planificación, etc) }\end{array}$} & A & ,284 & ,596 & 3,204 & 66 &, $002(* *)$ \\
\hline & B & & & 3,311 & 38,363 &, 002 \\
\hline \multirow{2}{*}{$\begin{array}{l}\text { Me inquieta la idea de que el Moodle reduzca el rol } \\
\text { del profesor, y sea negativo para la educación de los } \\
\text { estudiantes. }\end{array}$} & A & ,000 & ,988 & 1,146 & 63 &, 256 \\
\hline & B & & & 1,118 & 29,399 & ,273 \\
\hline \multirow{2}{*}{$\begin{array}{l}\text { Debería usar el Moodle en mis asignaturas pero no sé } \\
\text { cómo organizar y gestionar el aprendizaje de mis } \\
\text { estudiantes de esa manera }\end{array}$} & A & ,229 & ,634 &,- 019 & 62 & ,985 \\
\hline & $\mathrm{B}$ & & &,- 019 & 35,126 & ,985 \\
\hline
\end{tabular}

(nota: $* *=p<0,01 ; a=$ Se asumen varianzas iguales $\mathrm{y} b=$ No se asumen varianzas iguales)

El uso del Ambiente Virtual de Aprendizaje entre el profesorado de educación superior: un análisis de género. Annachiara Del Prete y Julio Cabero.

Página 9 de 20 
Como podemos observar los valores " $\mathrm{t}$ " obtenidos no permiten rechazar las $\mathrm{H} 0$ referidas a la existencia de diferencias significativas en la valoración de los obstáculos en función del sexo de los docentes, con un riesgo alfa de equivocarnos de $\mathrm{p}<0,05$; solamente se rechaza la $\mathrm{H} 0 \mathrm{y}$ se acepta la $\mathrm{H} 1$ con un riesgo alfa de equivocarnos de $\mathrm{p}<0,01$ en el obstáculo: "Creo que el Moodle es útil sólo para trabajo de administración y planificación docente (pruebas, planificación, etc), siendo dicho obstáculo más valorado por los profesores que por las profesoras.

\section{Dominio técnico, manejo didáctico y frecuencia de utilización de diferentes herramientas}

Con el objeto de analizar si había diferencias en función del género de los docentes respecto al dominio técnico, manejo didáctico y frecuencia de la utilización de diferentes herramientas ubicadas en la plataforma Moodle, aplicamos de nuevo el estadístico "t" de Student, alcanzado los valores que mostramos en la tabla $\mathrm{n}^{\mathbf{0}}$ 4. Para una correcta interpretación de los valores debe tenerse en cuenta que la escala de respuesta iba de 1 (muy poco) a 10 (muchísimo).

Tabla 4 Dominio Técnico, Dominio didáctico y Frecuencia de uso del AAI en función del género

\begin{tabular}{l|l|l|l|l|l|l|l|l|l}
\hline & Sexo & $\mathrm{N}$ & $\mathrm{M}$ & D.tp. & $\mathrm{F}$ & Sig & t & gl & Sig \\
\hline \multirow{2}{*}{ Dominio técnico } & $\mathrm{H}$ & 205 & 6,20 & 2,44 & 5,278 &, 022 &,- 789 & 567 &, 431 \\
\cline { 2 - 11 } & $\mathrm{M}$ & 364 & 6,38 & 2,69 & & & $\mathbf{- , 8 1 0}$ & $\mathbf{4 5 7 , 9 9 7}$ & $\mathbf{, 4 1 8}$ \\
\hline \multirow{2}{*}{ Manejo didáctico } & $\mathrm{H}$ & 200 & 5,99 & 2,37 & 4,072 &, 044 & 1,511 & 566 &, 131 \\
\cline { 2 - 11 } & $\mathrm{M}$ & 368 & 5,65 & 2,61 & & & $\mathbf{1 , 5 5 5}$ & $\mathbf{4 4 4 , 0 9 4}$ & $\mathbf{, 1 2 1}$ \\
\hline \multirow{2}{*}{ Frecuencia de uso } & $\mathrm{H}$ & 217 & 5,38 & 2,52 &, 051 &, 821 & $\mathbf{1 , 5 5 8}$ & $\mathbf{5 9 8}$ & $\mathbf{, 1 2 0}$ \\
\cline { 2 - 10 } & $\mathrm{M}$ & 383 & 5,05 & 2,50 & & & 1,555 & 446,487 &, 121 \\
\hline
\end{tabular}

Indicar, en primer lugar, que los valores medios alcanzados nos señalan niveles medios, tanto de dominio técnico, como de manejo didáctico y frecuencia de utilización. Por otra parte, señalar que los valores medios obtenidos no nos permiten rechazar ninguna de las $\mathrm{H} 0$ formuladas, con un riesgo alfa de equivocarnos de $\mathrm{p} \leq 0,05$; en consecuencia, podemos indicar que no hay diferencias significativas entre el dominio técnico y didáctico y la frecuencia de utilización de diferentes herramientas ubicadas en la plataforma Moodle de INACAP y el sexo del profesorado.

Especificamos que las herramientas sobre las que, específicamente, consultamos respecto al dominio técnico y didáctico y frecuencia de uso han sido: correo electrónico, foros, blog, wiki, tareas (entregas de archivos para calificación o retroalimentación), glosario, videos (youtube, vimeo, etc.), videoconferencia/audioconferencia, cuestionarios online, coevaluaciones (Tu evalúas a tus compañeros y ellos a ti), y consultas o encuestas. En la tabla n 5 presentamos las valoraciones medias y desviaciones típicas alcanzadas en cada uno de las herramientas y para las tres opciones demandas de respuestas requeridas.

Tabla 5 Uso de herramientas del AAI

\begin{tabular}{l|l|l|l|l}
\hline Herramienta & Sexo & D. técnico & M. didáctico & F. uso \\
\hline
\end{tabular}

El uso del Ambiente Virtual de Aprendizaje entre el profesorado de educación superior: un análisis de género. Annachiara Del Prete y Julio Cabero.

Página 10 de 20 
RED. Revista de Educación a Distancia. Núm. 62 Vol. 20. Artíc. 04, 31-03-2020

DOI: http://dx.doi.org/10.6018/red.400061

\begin{tabular}{|c|c|c|c|c|c|c|c|}
\hline & & $\mathrm{M}$ & D.tp. & $\mathrm{M}$ & D.tp. & $\mathrm{M}$ & D.tp. \\
\hline \multirow[t]{2}{*}{ Correo electrónico } & $\mathrm{H}$ & 9,13 & 1,520 & 8,52 & 1,978 & 9,15 & 1,782 \\
\hline & $\mathrm{M}$ & 9,34 & 1,429 & 8,13 & 2,543 & 8,81 & 2,154 \\
\hline \multirow[t]{2}{*}{ Foros } & $\mathrm{H}$ & 6,52 & 3,290 & 6,04 & 3,184 & 4,36 & 3,270 \\
\hline & $\mathrm{M}$ & 6,33 & 3,547 & 5,47 & 3,422 & 4,16 & 3,217 \\
\hline \multirow[t]{2}{*}{ Blog } & $\mathrm{H}$ & 4,10 & 3,133 & 3,88 & 3,129 & 2,37 & 2,341 \\
\hline & $M$ & 4,95 & 3,706 & 4,15 & 3,387 & 2,66 & 2,604 \\
\hline \multirow[t]{2}{*}{ Wiki } & $\mathrm{H}$ & 4,32 & 3,133 & 4,13 & 3,146 & 2,70 & 2,642 \\
\hline & $M$ & 4,80 & 3,677 & 4,10 & 3,408 & 2,60 & 2,568 \\
\hline \multirow{2}{*}{$\begin{array}{l}\text { Tareas (entregas de archivos para } \\
\text { calificación o retroalimentación) }\end{array}$} & $\mathrm{H}$ & 7,57 & 2,977 & 7,55 & 2,985 & 6,92 & 3,337 \\
\hline & $M$ & 7,70 & 3,086 & 7,27 & 3,171 & 6,62 & 3,326 \\
\hline \multirow[t]{2}{*}{ Glosario } & $\mathrm{H}$ & 5,19 & 3,371 & 4,84 & 3,342 & 3,57 & 3,249 \\
\hline & $M$ & 5,34 & 3,716 & 4,40 & 3,485 & 3,24 & 3,021 \\
\hline \multirow[t]{2}{*}{ Videos (youtube, Vimeo, etc.) } & $\mathrm{H}$ & 6,83 & 3,391 & 6,37 & 3,392 & 5,71 & 3,487 \\
\hline & $\mathrm{M}$ & 6,95 & 3,422 & 6,43 & 3,412 & 5,33 & 3,380 \\
\hline \multirow[t]{2}{*}{ Videoconferencia/Audioconferencia } & $\mathrm{H}$ & 3,83 & 3,339 & 3,62 & 3,216 & 2,32 & 2,506 \\
\hline & $\mathrm{M}$ & 4,78 & 3,658 & 4,05 & 3,380 & 2,68 & 2,721 \\
\hline \multirow[t]{2}{*}{ Cuestionarios online } & $\mathrm{H}$ & 4,89 & 3,546 & 4,77 & 3,538 & 3,16 & 3,110 \\
\hline & $\mathrm{M}$ & 5,27 & 3,855 & 4,75 & 3,701 & 3,36 & 3,226 \\
\hline \multirow{2}{*}{$\begin{array}{l}\text { Co-evaluaciones ( } \mathrm{Tu} \text { evalúas a tus } \\
\text { compañeros y ellos a ti) }\end{array}$} & $\mathrm{H}$ & 4,47 & 3,434 & 4,49 & 3,424 & 3,01 & 3,068 \\
\hline & $\mathrm{M}$ & 4,65 & 3,664 & 4,17 & 3,531 & 2,73 & 2,755 \\
\hline \multirow[t]{2}{*}{ Consultas o encuestas } & $\mathrm{H}$ & 5,07 & 3,531 & 4,92 & 3,475 & 3,58 & 3,249 \\
\hline & $\mathrm{M}$ & 5,38 & 3,721 & 4,76 & 3,594 & 3,62 & 3,212 \\
\hline
\end{tabular}

La tabla anterior nos permite indicar una serie de aspectos: el dominio técnico que indican los y las docentes es superior a su manejo didáctico, que salvo en el correo electrónico, la entrega de tareas, y la utilización de vídeos, el dominio, manejo y frecuencia de utilización por parte de los y las docentes es limitado. Por otra parte, y al analizar en qué herramientas y para qué dominio se podrían encontrar diferencias estadísticamente significativas en función del género de los docentes, aplicamos la "t" de Student; en este caso y para no extendernos, solamente presentaremos los casos en los cuales se han rechazado las H0 (tabla $\left.\mathrm{n}^{\mathrm{o}} 6\right)$.

Tabla 6 Dominio Técnico, Dominio didáctico y Frecuencia de uso de herramientas específicas de AAI

\begin{tabular}{l|l|l|l|l|l|l|l|l|l|}
\hline & $\mathrm{S}$ & $\mathrm{N}$ & $\mathrm{M}$ & D.tp. & $\mathrm{F}$ & Sig & t & gl & Sig \\
\hline \multirow{2}{*}{$\begin{array}{l}\text { Dominio técnico } \\
\text { Blog }\end{array}$} & $\mathrm{H}$ & 144 & 4,10 & 3,133 & 14,212 &, 000 & $\mathbf{- 2 , 3 2 5}$ & $\mathbf{4 1 8}$ & $\mathbf{, 0 2 1}(*)$ \\
\cline { 2 - 10 } & $\mathrm{M}$ & 276 & 4,95 & 3,706 & 14,212 &, 000 & $-2,325$ & 418 &, 021 \\
\hline \multirow{2}{*}{$\begin{array}{l}\text { Dominio didáctico } \\
\text { Correo electrónico }\end{array}$} & $\mathrm{H}$ & 196 & 8,52 & 1,978 & 9,883 &, 002 & 1,844 & 547 &, 066 \\
\cline { 2 - 10 } & $\mathrm{M}$ & 353 & 8,13 & 2,543 & & & $\mathbf{1 , 9 7 9}$ & $\mathbf{4 8 9}$ & $\mathbf{, 0 4 8}\left(^{*}\right)$ \\
\hline $\begin{array}{l}\text { Frecuencia de uso } \\
\text { Correo electrónico }\end{array}$ & $\mathrm{H}$ & 217 & 9,15 & 1,782 & 11,405 &, 001 & 1,976 & 590 &, 049 \\
\cline { 2 - 10 } & $\mathrm{M}$ & 375 & 8,81 & 2,154 & & & $\mathbf{2 , 0 7 7}$ & $\mathbf{5 2 0}$ & $\mathbf{0 3 8}(*)$ \\
\hline
\end{tabular}

Indicar que únicamente se han rechazado tres $\mathrm{H} 0$, que se referían al blog, en el caso del dominio técnico, y el correo electrónico en el dominio didáctico y de frecuencia de utilización, con un riesgo alfa de equivocarnos de $\mathrm{p}<0,05$; siendo en el primero de los casos superior en el caso de las profesoras, y en los dos siguientes superiores a los profesores. Al mismo tiempo hay que indicar que las mayores dispersiones en las respuestas se han dado en 
las profesoras que en los profesores, como podemos observar por las puntuaciones alcanzadas en las desviaciones típicas.

\section{Actividades realizadas con el AAI}

Otro de los objetivos pretendía analizar si había diferencias en la utilización de diferentes actividades llevadas a cabo en el AAI de INACAP. Entre los resultados obtenido indicar, en primer lugar, que en cerca del 50\% de las actividades ofrecidas, las/os docentes indicaron una utilización por debajo de la media de la escala de puntuación ofrecida; en concreto se obtuvo en: "Realizar Debates o foros de discusión", "Usar Simulaciones (uso de simuladores online)", "Entregar premios, puntuaciones, ranking (Metodología de Gamificación)", "Para desarrollar investigación", "Impulsar reflexión en los estudiantes a través de las distintas herramientas (wikis, foro, etc.)", "Utilizar herramientas para que los estudiantes debatieran", y "Para que los estudiantes realizaran ensayos."

Los valores alcanzados solamente nos permiten rechazar la $\mathrm{H} 0$ referida a la no existencia de diferencias significativas con un riesgo alfa de equivocarnos de $p \leq 0,05$, salvo en los casos de: "Realizar Debates o foros de discusión", "Usar Simulaciones (uso de simuladores online)" y "Utilizar rúbricas de evaluación". En los dos primeros casos al nivel de $\mathrm{p} \leq 0,05$ y en el segundo de los casos al de $\mathrm{p} \leq 0,01$. En el primero y tercero de los casos las puntuaciones mostradas por los profesores son superiores a las de las profesoras, mientras en el segundo de los casos, son las profesoras las que han encontrado puntuaciones superiores.

\section{Dominio del AAI}

Por lo que se refiere a sí se mostraron diferencias significativas entre los profesores y las profesoras en la autocalificación del dominio que tenían del AAI, puntuado de 1 a 10, indicar que los profesores obtuvieron una media de 6,57 con una desviación típica del 1.743 y las profesoras de 6,63 con una desviación típica del 1,934. Aplicada la "t” de Student para muestras independientes se obtuvo un valor de $-0,361$ que para 604 grados de libertad nos permitía obtener un nivel de significación del 0,725. Valor que no nos permitía rechazar la H0 que hacía referencia a la existencia de diferencias significativas en la calificación que hacían de su dominio del AAI los profesores y las profesoras.

\section{Discusión}

El estudio realizado nos permite obtener una serie de conclusiones respecto al objetivo general planteado que se refería a la posible existencia de diferencias en función del género del profesorado en la utilización del "Ambiente de Aprendizaje de INACAP", y de las diferentes herramientas en él incorporadas.

Como hemos podido observar los resultados obtenidos, y en particular en los análisis específicos respecto a frecuencia de uso, uso concreto de las herramientas, materiales movilizados, o actividades desarrolladas, no se evidencian diferencias significativas desde un punto de vista estadístico entre los profesores y las profesoras. Fijando nuestra atención en el propósito específico del estudio, observamos que, respecto al uso pedagógico y al uso de las diferentes herramientas del AAI, no se encuentran diferencias significativas entre las profesoras y los profesores encuestados.

El uso del Ambiente Virtual de Aprendizaje entre el profesorado de educación superior: un análisis de género. Annachiara Del Prete y Julio Cabero.

Página 12 de 20 
De los análisis llevados a cabo, podemos afirmar que la mayoría de las y los docentes encuestados si hace uso del AAI, si bien son los profesores más que las profesoras, y ambos reconocen realizar una baja explotación de las diferentes actividades didácticas que el Ambiente Virtual de Aprendizaje ofrece. Esta tendencia responde a lo que se indica en diferentes investigaciones (Buabeng-Andoh, 2012; Raman, Rozalina Khalid, \& Rizuan 2014; Teo, 2011) y viene confirmada por otro resultados que se observa en nuestros datos, en los cuales se destaca que, si bien las y los docentes indican presentar un buen dominio técnicos de las herramientas, los dos colectivos afirman no poseer un alto dominio por lo que respecta el uso pedagógico de las mismas.

Siguiendo con la discusión de los resultados, se observa un menor uso técnico de los Blog por parte de las profesoras; al mismo tiempo las profesoras declaran utilizar en mayor frecuencia, respecto a los profesores, el correo electrónico por fines didáctico, tendencia ésta última, que confirmaría una actitud más activa de las profesoras hacia la enseñanza y al aprendizaje en ambiente virtuales (González-Gómez et al 2012; Egbo, Chinwe, Ikechukwu, \& Onwumere 2011; Suri y Sharma 2013;). Un resultado a destacar es la ausencia de diferencias significativas en las habilidades técnicos pedagógicas, así como en la auto calificación del dominio del AAI entre profesoras y profesores, hecho que nos indica un cambio, ya adelantado por la literatura, hacia una paridad de habilidades y competencias digitales, (Chou, Wu, \& Chen, 2011; Schumacher \& Morahan-Martin, 2001; Teo, 2010) y un aumento de percepción de habilidades por parte de las mujeres, cambio que se confirma en nuestro estudio.

\section{Conclusiones}

Nuestro trabajo apunta con claridad a que la brecha digital de género se está reduciendo, y podríamos decir que, no existe en el contexto de formación universitario estudiado. Hecho que está en consonancia con los resultados encontrados en las últimas investigaciones donde el género del docente no es predictor de la frecuencia de utilización de las tecnologías (Chou, Wu \& Chen 2011; Güdüz \& İşman, 2018; Lohbeck, Hagenauer \& Frenzel, 2018; Ríos, Gómez \& Priscilla, 2018); resultados que también empiezan a encontrarse entre estudiantes universitarios (Vázquez-Cano, Meneses \& García-Garzón, 2017).

Estos resultados llevan a plantearnos dos aspectos: por una parte, la necesidad de seguir estableciendo planes de formación de profesorado para la utilización de las TIC (Kafyulilo, et al. 2016; Villalba, González-Rivera \& Diaz-Pulido, 2017), y por otra, la necesidad de que la misma se enfoque desde planteamientos diferentes a los que se han llevado hasta el momento (Cabero \& Barroso, 2016). Se destacan diferencias solamente en aspectos puntuales, de las distintas dimensiones analizadas, entre la cuales encontramos una tendencia más alta de los hombres respecto a las mujeres, en considerar la AAI prevalentemente un instrumento de gestión y planificación docente, resultado que coincide parcialmente con otras investigaciones (González et al. 2012; Kim, 2010; Ong y Lai, 2006). Siempre los profesores presentan una mayor frecuencia de uso de la plataforma para requerir el envío de trabajo y para el uso de rúbrica de evaluación, y esto vendría a confirmar una mayor tendencia de los profesores al uso del AAI para gestión de la docencia.

Contrariamente a lo que se observa en las investigaciones analizadas (Asiri 2012; Casquero, García y González 2014) los datos de nuestro estudio arrojan una frecuencia mayor, por parte

El uso del Ambiente Virtual de Aprendizaje entre el profesorado de educación superior: un análisis de género. Annachiara Del Prete y Julio Cabero.

Página 13 de 20 
de los profesores, en el uso de debates y foros de discusiones, así como un mayor uso del power-point. Generalmente las mujeres suelen presentar mayor disposición al uso de herramientas de comunicación en los AAI que los hombres (Kim 2010; Kirkup y Kirkwood 2005).

Nuestro estudio aporta indirectamente una velada crítica al imaginario social que se ha creado respecto a que el profesorado, por nacer en un momento histórico determinado, se definen inmigrantes digitales y por ello menos expertos en tecnología que el alumnado, considerado como nativo digital. Por el contrario, nuestro profesorado no percibe como obstáculo para utilizar el AAI el no "creerse capaz de usar Moodle de manera efectiva en su labor docente". Dato que va en consonancia con lo alcanzado en diferentes investigaciones que ya no apuntan tales diferencias entre docentes y discentes (Castellanos, Sánchez \& Calderero, 2017; Lai \& Hong, 2015; Jelfs \& Richardson, 2013; Wang, Hsu, Campbell, Coster \& Longhurst, 2014). Finalmente, a la luz de los datos analizados podemos confirmar lo que la literatura viene sosteniendo en los últimos años, o sea, que la diferencia entre hombres y mujeres en las habilidades digitales y uso de la tecnología en el contexto educativo resulta ser siempre menos significativa. Puede que hoy en día, todavía se den mayor percepción de habilidades digitales por parte los hombres respecto a las mujeres, sin embargo, en la práctica hemos visto que ya no se presentan diferencias en el manejo técnico y en el uso didáctico de las TIC. Como líneas futuras de investigación creemos que sería interesante replicar la investigación en otro contexto universitario chileno, replicarla en otro contexto universitario latinoamericano, avanzar en estudios de carácter tecnofeministas sobre usos específicos que las profesoras hacen de las tecnologías, así como a investigar sobre tipologías de usos didácticos que hacen de las mismas.

\section{Declaración}

El autor no informó ningún potencial conflicto de intereses.

Presentación del artículo: 10 de enero de 2020 Fecha de aprobación: 27 de febrero de 2020 Fecha de publicación: 31 de marzo de 2020

Del Prete, A., y Cabero, J. (2020). El uso del Ambiente Virtual de Aprendizaje entre el profesorado de educación superior: un análisis de género. RED. Revista de Educación a Distancia, 62. Consultado el (dd/mm/aaaa) en http://dx.doi.org/10.6018/red.400061

\section{Financiación}

Esta investigación no ha recibido ninguna subvención específica de los organismos de financiación en los sectores públicos, comerciales o sin fines de lucro. 


\section{Referencias bibliográficas}

Ahmad, T. B. T., Basha, K. M., Marzuki, A. M., Hisham, N. A. \& Sahari, M. (2010). Faculty's acceptance of computer based technology: Cross-validation of an extended model. Australasian Journal of Educational Technology, 26(2), 268-279. http://www.ascilite.org.au/ajet/ajet26/ahmad.html.

Alaminos, A. (2006). El muestreo en la investigación social. En A. Alaminos y J.L. Castejón, Elaboración, análisis e interpretación de encuestas, cuestionarios y escalas de opinión (46-67). Alcoy: Marfil.

Almerich Cerveró, G., Suárez Rodríguez, J. M., Jornet Meliá, J. M., \& Orellana Alonso, M. N. (2011). Las competencias y el uso de las Tecnologías de Información y Comunicación (TIC) por el profesorado: estructura dimensional. Revista electrónica de investigación educativa, 13(1), 28-42.

Aristovnik, A., Tomazevic, N., Kerzic, D., \& Umek, L. (2017). The impact of demographic factors on selected aspects of e-learning in higher education. The International Journal of Information and Learning Technology, 34(2), 114-12

Asiri, M. J. S. (2012). Factors influencing the use of learning management system in Saudi Arabian Higher Education: A theoretical framework. Higher Education Studies, 2(2), 125137.

Bandura, A. (1977). Self-efficacy: Toward a Unifying Theory of Behavioural Change. Psychological Review, 84 (2), pp. 191-215. http://dx.doi.org/10.1037/0033295X.84.2.191

Bandura, A., Barbaranelli, C., Caprara, G. y Pastorelli, C. (2001). Self-efficacy Beliefs as Shapers of Children's Aspirations and Career Trajectories. Child Development, 72 (1), pp. 187-206. http://dx.doi.org/10.1111/1467-8624.00273

Bidarian, S., Davoudi, A.M. (2011) A Model for application of ICT in the process of teaching and learning. International Conference on Education and Educational Psychology (ICEEPSY). Procedia - Social and Behavioral Sciences 29 (2011) 1032-1041.

Bishop, Y.M., Fienberg, S.E. y Holland, P.W. (2007). Discrete Multivariate Analysis. Theory and Applications. New York: Springer.

Buabeng-Andoh, C. (2012). Factors influencing teachers' adoption and integration of information and communication technology into teaching: A review of the literature. International Journal of Education and Development using Information and Communication Technology, 8(1), 136.

Cabero J. y Barroso, J. (2016). ICT teacher training: a view of the TPACK model. Cultura $y$ Educación, 28(3), 633-663. http://dx.doi.org/10.1080/11356405.2016.1203526.

Cabero, J., Sampedro, B y Gallego, O. (2016). Valoraciones de la "aceptación de la tecnología de formación virtual" por profesores universitarios asistentes a un curso de formación virtual. EDUTEC, Revista Electrónica de Tecnología Educativa, 56, 31-47. Recuperado el 27/02/2018 de http://www.edutec.es/revista.

El uso del Ambiente Virtual de Aprendizaje entre el profesorado de educación superior: un análisis de género. Annachiara Del Prete y Julio Cabero.

Página 15 de 20 
Cantón, I., Cañón, R y Grande, M. (2017). La comunicación como subdimensión de la competencia digital en futuros maestros de primaria. Pixel-Bit. Revista de Medios y Educación, 50, 33-47.

Casquero, G. B., García, L. M. C., \& González, R. F. L. (2014). Competencias Tecnológicas de los profesores de Infantil y Primaria de Extremadura en función del género. IE Comunicaciones: Revista Iberoamericana de Informática Educativa, (19), 3.

Castellanos, A., Sánchez, C. y Calderero, J. (2017). Nuevos modelos tecnopedagógicos. Competencia digital de los alumnos universitarios. Revista Electrónica de Investigación Educativa, 19(1), 1-9, (Recuperado de http://redie.uabc.mx/redie/article/view/1148

Clark, R. C. and Meyer, R. E. (2011), E-learning and the science of instruction: proven guidelines for consumers and designers of multimedia learning, Pfeiffer, New York.

Chen, R. (2010). Investigating models for preservice teachers' use of technology to support student-centered learning. Computers \& Education, 55(1), 32-42.

Chiu, J. L., \& Linn, M. C. (2012). The role of self-monitoring in learning chemistry with dynamic visualizations. In Zohar, A., Dori, Y. J. (Eds), Metacognition in science education: Trends in current research. 133-163. Dordrecht: Springer.

Chou, C., Wu, H. C., \& Chen, C. H. (2011). Re-visiting college students' attitudes toward the Internet-based on a 6-T model: Gender and grade level difference. Computers \& Education, 56(4), 939-947.

Dusick, D. M. (1998). What social cognitive factors influence faculty members' use of computers for teaching? A literature review. Journal of Research on Computing in Education, 31 (2), 123-137.

Egbo O. P., Chinwe R., Ikechukwu C., \& Onwumere J. U. (2011). Gender perception and attitude towards ELearning: A case of Business students, University of Nigeria. International Journal of Computer Application, 1, 135-148.

Fernández, M. y Vázquez, S. (2016). La larga y compleja marcha del CLIP al CLIP. Escuela y profesorado en el nuevo entrono digital. Madrid: Fundación Telefónica-Ariel.

González-Gómez, F., Guardiola, J., Rodríguez, Ó. M., \& Alonso, M. Á. M. (2012). Gender differences in e-learning satisfaction. Computers \& Education, 58(1), 283-290.

Grundy, F. (1996) Women and computers. Exeter, England: Intellect Books

Güdüz, A. e İşman, A. (2018). Pre-Service Teachers' Perception of Distance Education. TOJET: The Turkish Online Journal of Educational Technology, 17(1), 125-129.

Jelfs, A. y Richardson, J. (2013). The use of digital technologies across the adult life span in distance education. British Journal of Educational Technology, 44, 2, 338-351. doi:10.1111/j.1467-8535.2012.01308.x./s11423-014-9355-4.

Kafyulilo, A., Fisser, P., \& Voogt, J. (2016). Factors affecting teachers' continuation of technology use in teaching. Education and Information Technologies, 21(6), 1535-1554. doi:10.1007/s10639-015-9398-0

El uso del Ambiente Virtual de Aprendizaje entre el profesorado de educación superior: un análisis de género. Annachiara Del Prete y Julio Cabero.

Página 16 de 20 
Kim, Y. L. (2010). Gender role and the use of university library website resources: a social cognitive theory perspective. Journal of Information Science, 36(5), 603-617.

Kirkup, G., \& Kirkwood, A. (2005). Information and communications technologies (ICT) in higher education teaching - a tale of gradualism rather than revolution. Learning, Media and Technology, 30(2), 185-199.

Koch, S., Muller, S. \& Sieverding, M. (2008). Women and computers. Effects of stereotype threat on attribution of failure. Computers \& Education, 51(4), 1795-1803. http://dx.doi.org/10.1016/j.compedu.2008.05.007

Krause, M., Pietzner, V., Dori, Y. J., \& Eilks, I. (2017). Differences and Developments in Attitudes and Self-Efficacy of Prospective Chemistry Teachers Concerning the Use of ICT in Education. Eurasia Journal of Mathematics, Science and Technology Education, 13(8), 4405-4417.

Lai, K. y Hong, K. (2015). Technology use and learning characteristics of students in higher education: Do generational differences exist? British Journal of Educational Technology, 46(4), 725-738. doi:10.1111/bjet.12161.

Law, N., Pelgrum, W. J., \& Plomp, T. (Eds.). (2008). Pedagogy and ICT use in schools around the world: Findings from the IEA SITES 2006 study (Vol. 23). Springer Science $\&$ Business Media

Lohbeck, A., Hagenauer, G., \& Frenzel, A. C. (2018). Teachers' self-concepts and emotions: Conceptualization and relations. Teaching and Teacher Education, 70, 111-120.

Mahmud, R., \& Ismail, M. A. H. (2010). Impact of Training and Experience in Using ICT on in-Service Teachers' Basic ICT Literacy. Malaysian Journal of Educational Technology, 10 (2), 6.

Mas, X. (2017). El tejido de Weiser. Claves, evolución y tendencias de la educación digital. Barcelona: OuterEDU.

Moran, M., Hawkes, M., \& El Gayar, O. (2010). Tablet personal computer integration in higher education: Applying the unified theory of acceptance and use technology model to understand supporting factors. Journal of Educational Computing Research, 42(1), 79101. http://dx.doi.org/10.2190/EC.42.1.d

O’Dwyer, L. y Bernauer, J. (2014). Quantitative research for the qualitative researcher. California: Sage.

OCDE (2010). PISA 2009 at a glance. OECD Publishing http://dx.doi.org/10.1787/9789264095298-en.

Ong, C. S. \& Lai, J. Y. (2006). Gender differences in perceptions and relationships among dominants of e-learning acceptance. Computers in Human Behavior, 22(5), 816-829. http://dx.doi.org/10.1016/j.chb.2004.03.006

Pohl, M. (1997). The Internet: a feminine technology? In R. Lander, \& A. Adam (Eds.), Women in Computing (pp. 190-197). Exeter, England: Intellect Books.

El uso del Ambiente Virtual de Aprendizaje entre el profesorado de educación superior: un análisis de género. Annachiara Del Prete y Julio Cabero.

Página 17 de 20 
Radovan, M. y Kristl, N. (2017). Acceptance of Technology and its Impact on Teacher's Activities in Virtual Classroom: Integrating UTAUT and CoI into a Combined Model. TOJET: The Turkish Online Journal of Educational Technology, 16(3), 11-22.

Raman A., Rozalina Khalid Y., \& Rizuan M. (2014). Usage of learning management system (Moodle) among postgraduate students: UTAUT model. Asian Social Science, 10, 186195 http://dx.doi.org/10.5539/ass.v10n14p186.

Ríos, J.M., Gómez, E. y Priscilla, M. (2018). Valoración de competencias TIC del profesorado universitario: un caso en Chile. Pixel-Bit. Revista de Medios y Educación, 52, 55-65. doi: http://dx.doi.org/10.12795/pixelbit.2018.i52.04.

Rodríguez Espinosa, H., Restrepo Betancur, L. F., \& Aranzazu Taborda, D. (2016). Desarrollo de habilidades digitales docentes para implementar ambientes virtuales de aprendizaje en la docencia universitaria. Sophia, 12(2), 261-270.

Rodríguez, E., Restrepo, L. y Aranzazu, D. (2014). Alfabetización informática y uso de sistemas de gestión del aprendizaje (LMS) en la Docencia. Revista de Educación Superior, $43,171,139-159$.

Rodríguez-García, A., Raso, F. y Ruiz-Palmero, J. (2019). Competencia digital, educación superior y formación del profesorado: un estudio de meta-análisis en la web of science, 54, 65-82, DOI: https://doi.org/10.12795/pixelbit.2019.i54.04.

Romero, R., Cabero, J. y Barroso, J. (2016). E- Learning of Andalusian University's Lecturers. Gender. TOJET: The Turkish Online Journal of Educational Technology, 15, $2,25-37$.

Rossi Cordero, A. E., \& Barajas Frutos, M. (2015). Elección de estudios CTIM y desequilibrios de género. Enseñanza de las ciencias, 0059-76.

Sabariego, M. (2012). El proceso de investigación (parte 2). En Bisquerra, R. (coord.), Metodología de la investigación educativa (pp. 127-163). (3ª ed.). Madrid: La Muralla.

Schumacher, P., \& Morahan-Martin, J. (2001). Gender, Internet and computer attitudes and experiences. Computers in Human Behavior, 17(1), 95-110.

Sieverding, M. \& Koch, S. C. (2009). (Self-)Evaluation of computer competence: How gender matters. Computers \& Education, 52(3), 696-701. http://dx.doi.org/10.1016/j.compedu.2008.11.016

Sosa, E., Salinas, J., \& De Benito, B. (2018). Factors that facilitate or limit the incorporation of emerging technologies in the classroom. International Online Journal of Education and Teaching (IOJET), 5(1), 38-59.

Suárez, J., Almerich, G. Gargallo, B. y Aliaga, F. (2013). Las competencias del profesorado en TIC: estructura básica. Educación XX1, 16, 1, 39-62. DOI: 10.5944/educxx1.16.1.716.

Suri G., \& Sharma S. (2013). The impact of gender on attitude towards computer technology and E-learning: An exploratory study of Punjab University, India. International Journal of Engineering Research, 2, 132-136.

El uso del Ambiente Virtual de Aprendizaje entre el profesorado de educación superior: un análisis de género. Annachiara Del Prete y Julio Cabero.

Página 18 de 20 
Sussman, N., \& Tyson, D. (2000). Sex and power: gender differences in computer mediated interactions. Computers in Human Behavior, 16(4), 381-394.

Teo, T. (2010). Measuring the effect of gender on computer attitudes among pre-service teachers: A multiple indicators, multiple causes (MIMIC) modeling. Campus-Wide Information Systems, 27(4), 227-239. http://dx.doi.org/10.1108/10650741011073770

Teo, T. (2011). Factors influencing teachers' intention to use technology: Model development and test. Computers \& Education, 57(4), 2432-2440.

Terzis, V. \& Economides, A. A. (2011). The acceptance and use of computer based assessment. Computers \& Education, 56(4), 1032-1044. http://dx.doi.org/10.1016/j.compedu.2010.11.017

Tsai, P.S., \& Tsai, C. C. (2010). Elementary school students' attitudes and self-efficacy of using PDAs in a ubiquitous learning context. Australasian Journal of Educational Technology, 26 (3), 297-308

Tüfekçi Z. (2008). Gender, social capital and social network(ing) sites: Women bonding, men searching. Boston: Annual meeting of the American Sociological Association. Sheraton Boston and the Boston Marriott Copley Place.

Turkle, S. (1995). Life on the screen: identity in the age of the Internet. New York: Simon \& Schuster.

United Nations (2014). Measuring ICT and gender: An assessment .New York and Geneva: United Nations Retrievedfrom http://unctad.org/en/PublicationsLibrary/webdtlstict2014d1_en.pdf.

Vázquez-Cano, E., Meneses, E. L., \& García-Garzón, E. (2017). Differences in basic digital competences between male and female university students of Social Sciences in Spain. International Journal of Educational Technology in Higher Education, 14(1), 27

Vekiri, I., \& Chronaki, A. (2008). Gender issues in technology use: Perceived social support, computer self-efficacy and value beliefs, and computer use beyond school. Computers \& Education, 51 , 1392-1404. http://dx.doi.org/10.1016/j.compedu.2008.01.003

Venkatesh, V., \& Morris, M. G. (2000). Why don't men ever stop to ask for directions? Gender social influence and their role in technology acceptance and usage behaviour. MIS Quarterly, 24 (1), 115-139. http://dx.doi.org/10.2307/3250981

Villalba, A., González-Rivera, M. D., \& Diaz-Pulido, D. (2017). Obstacles Perceived by Physical Education Teachers to Integrating ICT. TOJET: The Turkish Online Journal of Educational Technology, 16(1), 83-92.

Wang, Sh., Hsu, H., Campbell, T., Coster D. \& Longhurst, M. (2014). An investigation of middle school science teachers and students use of technology inside and outside of classrooms: considering whether digital natives are more technology savvy than their teachers. Education Tech Research Dev, 62:637-662. DOI 10.1007

El uso del Ambiente Virtual de Aprendizaje entre el profesorado de educación superior: un análisis de género. Annachiara Del Prete y Julio Cabero.

Página 19 de 20 
Weiser, E. (2000). Gender differences in Internet use patterns and Internet application preferences: a two sample comparison. CyberPsychology and Behavior, 3(2), 167-178.

Wong, K., Teo, T., \& Russo, S. (2012). Influence of gender and computer teaching efficacy on computer acceptance among Malaysian student teachers: An extended technology acceptance model. Australasian Journal of Educational Technology, 28(7), 1190-1207.

Zeldin, A. L. y Pajares, F. (2000). Against the Odds: Self-efficacy Beliefs of Women in Mathematical, Scientific and Technological Careers. American Educational Research Journal, 37(1), pp. 215-246. http://dx.doi.org/10.3102/00028312037001215.

El uso del Ambiente Virtual de Aprendizaje entre el profesorado de educación superior: un análisis de género. Annachiara Del Prete y Julio Cabero. 